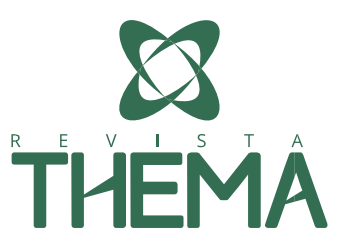

\title{
O desenvolvimento da Cognição Numérica: compreensão necessária para o professor que ensina Matemática na Educação Infantil
}

\section{The development of Numerical Cognition: a necessary understanding for the teacher who teaches Mathematics in Preschool}

Sidney Lopes Sanchez Júnior ${ }^{1}$; Marília Bazan Blanco²

\section{RESUMO}

A Matemática está presente no universo da criança desde o seu nascimento. Diferentes abordagens procuram entender como se dá o desenvolvimento do conceito de número e das habilidades numéricas na criança, e o presente artigo de revisão tem por objetivo discutir como se desenvolve a Cognição Numérica, a partir da perspectiva da Psicologia Cognitiva e da Neurociência, e a importância deste conhecimento para melhorar a prática do professor que atua no ensino da Matemática com crianças pré-escolares. A partir desta perspectiva, entende-se que a Matemática mais complexa e abstrata evolui de uma mais simples, de origem biológica. A Cognição Numérica é influenciada por fatores biológicos, cognitivos, educacionais e culturais, sendo que o Senso Numérico inato, constitui o sistema primário, e o sistema secundário é formado pelo Processamento Numérico (Compreensão e Produção Numérica) e Cálculo. Quando o professor da Educação Infantil tem ciência de como essa cognição numérica se desenvolve, torna-se capaz de pensar em atividades que possam favorecer esse desenvolvimento.

Palavras-chave: Cognição Numérica; Matemática; Educação Infantil.

\begin{abstract}
Mathematics has been present in the child's universe since birth. Different approaches seek to understand how the concept of number and numerical abilities develops in children, and the present review article aims to discuss how Numerical Cognition develops, from the perspective of Cognitive Psychology and Neuroscience, and the importance of this knowledge to improve the practice of the teacher who works in the teaching of Mathematics with preschool children. From this perspective, it has understood that the more complex and abstract Mathematics evolves from a simpler one, of biological origin. Numerical Cognition is influenced by biological, cognitive, educational and cultural factors, and innate Number Sense constitutes the primary system, and Numerical Processing (Numerical Understanding and Production) and Calculus form the secondary system. When the Early Childhood teacher is aware of how this numerical cognition develops, becomes more apt to think of activities that may favor his development.
\end{abstract}

Keywords: Numerical Cognition; Mathematics; Child education.

\footnotetext{
${ }^{1}$ Prefeitura Municipal de Cornélio Procópio, Cornélio Procópio/PR - Brasil.

2 UENP - Universidade Estadual do Norte do Paraná, Cornélio Procópio/PR - Brasil.
} 


\section{INTRODUÇÃO}

Desde o nascimento, as crianças já estão inseridas em um universo onde os conhecimentos matemáticos estão presentes (Brasil, 1998), sendo o envolvimento com números algo natural. 0 contato com a Matemática fora da escola costuma ser prazeroso e as crianças se divertem com brincadeiras que envolvem números. No entanto, a aprendizagem da Matemática na escola parece já não ser mais tão divertida (Lorena, Castro-Caneguim, Carmo, 2013).

De acordo com os dados da Avaliação Nacional da Alfabetização de 2014, o desempenho de 57\% dos estudantes brasileiros em Matemática concentra-se nos dois primeiros níveis, considerados muito básicos, sendo que $24 \%$ encontram-se no Nível 1, que representa uma aprendizagem ainda incipiente, indicando que as crianças ainda não dominam os conceitos esperados. Apenas $25 \%$ encontram-se no Nível 4, considerado o nível mais adequado (Qedu, 2017). Já os resultados da Prova Brasil de 2015, afirmam que $8 \mathrm{em}$ cada 10 crianças que terminaram o ensino fundamental não aprenderam o adequado em Matemática. Os dados revelam que apenas 39\% dos alunos matriculados na rede pública apresentam um aprendizado adequado (Qedu, 2017).

As dificuldades de aprendizagem na Matemática podem estar relacionadas a diversos fatores, desde problemas no Sistema Nervoso Central, com falhas no processamento e no armazenamento de informações, ou privações socioeconômicas e culturais e problemas pedagógicos, em particular no modo como se ensina a Matemática (Ciasca, 2004).

Sabe-se que mesmo as crianças mais novas possuem uma certa predisposição para lidar com quantidades, denominada senso numérico, e que problemas no senso numérico podem resultar em dificuldades no desenvolvimento do raciocínio matemático posterior e na aprendizagem da Matemática formal (Corso, Dorneles, 2010).

O desenvolvimento do raciocínio matemático pela criança, desde o senso numérico (sistema primário) até a aprendizagem da Matemática formal (sistema secundário), assim como a área da Neurociência e da Psicologia Cognitiva que estuda esse desenvolvimento, tem sido denominada de Cognição Numérica (Santos et al., 2016), e acredita-se que sua compreensão seja de extrema importância para o professor que ensina Matemática na Educação Infantil e Ensino Fundamental.

Assim, o objetivo deste artigo é discutir como se dá o desenvolvimento da cognição numérica nas crianças, tornando essa abordagem acessível ao professor que ensina Matemática na Educação Infantil e Ensino Fundamental. Para tanto, serão apresentados os conceitos de habilidades matemáticas primárias e secundárias (Geary, 2000), Senso Numérico (Dehaene, 1997), o modelo de McCloskey, Caramazza e Basili (1985) de processamento numérico e cálculo, o Modelo de Desenvolvimento da Cognição Numérica de von Aster e Shalev (2007), o Modelo do triplo código de Stanilas Dehaene (1992) e Modelo de processamento de informação aritmética de Menon (2010). 


\section{O DESENVOLVIMENTO DAS HABILIDADES NUMÉRICAS}

Segundo Geary (2000), as habilidades matemáticas humanas são divididas em habilidades primárias e secundárias. As habilidades primárias envolvem uma compreensão implícita de numerosidade, ordinalidade, início da contagem e aritmética simples, de origem biológica e que se desenvolve gradualmente durante os anos pré-escolares, juntamente com a linguagem. Já as habilidades secundárias são determinadas culturalmente, pelo sistema de ensino, e envolvem o conceito de número e a contagem, a aritmética, cálculo e resolução de problemas escritos.

Nessa perspectiva, entende-se que a denominada Cognição Numérica seja influenciada por fatores biológicos, cognitivos, educacionais e culturais, e se constitui de um sistema primário, o Senso Numérico, e sistemas secundários como o Processamento Numérico, o qual se subdivide em Compreensão Numérica (entendimento dos símbolos numéricos) e Produção Numérica (leitura, escrita e contagem de números) e o Cálculo (operações matemáticas) (Molina, Ribeiro, Santos, von Aster, 2015).

\subsection{Habilidades matemáticas primárias e o senso numérico}

De acordo com Dehaene (1997), o senso numérico (do Inglês, number sense) foi definido por Tobias Dantzig, em 1954, como uma faculdade inata que permite ao homem discriminar a retirada ou adição de elementos num pequeno grupo. Nessa época, a teoria Piagetiana, que negava que as crianças pequenas possuíssem habilidades matemáticas, era considerada a mais aceita na Psicologia, e demorou mais de 20 anos para que ela fosse refutada e a existência do senso numérico fosse confirmada.

Dehaene (2001) afirma que o senso numérico é a capacidade do indivíduo de compreender rapidamente, aproximar e manipular quantidades numéricas. É a capacidade mais básica e inata de reconhecer, representar, comparar, estimar, julgar magnitudes não verbais, somar e subtrair números sem a utilização de recursos de contagem, presente em todo ser humano, ainda em seu primeiro ano de vida, e também em alguns animais (Dehaene, 2001). Seria composto pela subitização e aproximação de grandes numerosidades ou estimativa (Dehaene, 1997)

A subitização (tradução do Inglês, subitizing, "de súbito") se refere à capacidade de discernir rapidamente o número de um conjunto com até quatro elementos (Lakoff, Nunez, 2000) e de responder diferencialmente ao acréscimo ou retirada de elementos nesse conjunto (Lorena, CastroCaneguin, Carmo, 2013).

Vários estudos descritos na literatura sugerem que a subitização tem base filogenética, estando presente em bebês humanos e animais (para revisões, ver Dehaene, 1997, Geary, 2000; Barbosa, 2007; 2012; Blanco et al., 2012; Marcilese, 2012).

Por meio desses estudos, pode-se identificar, por exemplo, que recém-nascidos conseguem discriminar numerosidade de pequenos grupos com dois e três elementos (Antell, Keating, 1983) e que bebês de cinco meses são capazes de realizar cálculos simples (soma e subtração com dois ou três elementos) (Wynn, 1992). Resultados semelhantes foram descritos com macacos rhesus (Hauser, Macneilage, Ware, 1996) e cães (West, Young, 2002). 
Quando o número de elementos ultrapassa quatro, a subitização é substituída pela estimativa. Segundo Hauser e Spelke (2004), bebês humanos, assim como outros animais, são capazes de discriminar diferenças em conjuntos de objetos, de forma aproximada, principalmente quando houver bastante discrepância entre os conjuntos (limite de Weber ${ }^{3}$ ).

Um senso numérico bem desenvolvido é refletido na habilidade da criança de estimar quantidade, reconhecer erros de julgamento de magnitude ou de medida, fazer comparações quantitativas do tipo maior do que, menor do que, e equivalência. Crianças com senso numérico desenvolvido sabem o que os números significam (Corso, Dorneles, 2010, p.299).

Embora existam evidências da base inata do senso numérico, Barbosa (2007; 2012), Corso e Dorneles (2010) e Marcilese (2012) discutem que ainda não há consenso entre os pesquisadores, uma vez que alguns estudos realizados com bebês apresentaram resultados conflitantes. De acordo com Barbosa (2012), embora não haja dúvidas de que os bebês sejam sensíveis às informações numéricas presentes no ambiente, ainda existem várias questões pendentes quanto à origem dessa sensibilidade.

Grande parte dos estudos que apontam para a existência de um senso numérico inato nos bebês utilizam-se do paradigma da habituação/novidade (segundo o qual os bebês têm preferência pela novidade) e pelo paradigma da violação da expectativa, defendendo que as crianças tendem a olhar por mais tempo para eventos inesperados (Marcilese, 2012).

Corso e Dorneles (2010) apontam que tais estudos sofrem críticas pela falta de precisão em identificar a variável que realmente controla o comportamento dos bebês, se seria a numerosidade ou outras varáveis como brilho, densidade ou perímetro dos objetos utilizados. Ainda, de acordo com Barbosa (2012), outros estudos realizados sugeriram que os bebês apresentam preferência por variáveis perceptivas, e não numéricas. Como exemplo, Clearfield e Mix (1999), testando bebês de seis a oito meses, identificaram que estes ficam sob controle do contorno da área do conjunto, e não do número de elementos que esse conjunto possui. Assim, embora as crianças pareçam discriminar a quantidade, elas estão apenas percebendo "a diferença entre os contornos gerais, da área ou massa dos conjuntos de objetos" (Papalya, Olds, Feldman, 2010, p.199).

Portanto, alguns autores discutem que os bebês possuem apenas habilidades perceptivas mais gerais que podem levá-los a focalizar a atenção aos aspectos numéricos, e não um módulo inato especificamente numérico (Barbosa, 2012). Ou seja, quando durante os experimentos ele é exposto várias vezes aos mesmos estímulos, pode começar a prestar atenção nos aspectos numéricos desses estímulos, algo que até então ele não fazia. O fato é que, mesmo que se admita a fragilidade de alguns desses estudos, já é sabido que os bebês apresentam mais conhecimento numérico, e bem mais cedo, do que Piaget Ihes atribuiu (Cole, Cole, 2003).

Geary (2000) utiliza a terminologia habilidades quantitativas primárias no sentido semelhante ao senso numérico. Segundo ele, essas habilidades relacionam-se a numerosidade (habilidade para determinar precisamente a quantidade de elementos em grupos de até quatro elementos, sem contagem), ordinalidade (entendimento de mais e menos, por exemplo, "de que 3 vale mais que 2", e que anteriormente à contagem, se restringe a números menores que cinco), contagem (noção de

${ }^{3}$ Limite ou Discriminação de Weber; quanto maior a diferença entre as numerosidades, maior a probabilidade de uma discriminação correta. (Blanco et al, 2012) 
ordenar e seriar, e, após o desenvolvimento da linguagem, relacionar os números aos objetos) e aritmética simples (sensibilidade ao aumento ou diminuição de itens em um pequeno grupo).

Segundo Geary (2000), as habilidades primárias formariam a estrutura necessária para o desenvolvimento do conceito de número, a contagem e a aritmética. A partir dessa habilidade mais básica dos bebês (senso numérico), ainda na idade pré-escolar, as crianças começam a ter contato com as palavras relacionadas à contagem, e, como já possuem uma noção de ordinalidade, iniciam o processo de contar (embora esse processo ainda não esteja maduro e as crianças cometam muitos erros). Assim, o sistema numérico pré-verbal se torna integrado à linguagem, resultando na contagem verbal.

\subsection{Habilidades matemáticas secundárias}

De acordo com Geary (2000), as habilidades secundárias são dependentes da escolarização, e incluem o conceito de número e contagem e a aritmética (cálculo e problemas verbais).

O conceito de número e a contagem, na escola primária, incluem o entendimento do sistema de base 10 e a utilização e transformação dos diferentes tipos de representação numérica (verbal/arábico). Nessa fase, é comum que as crianças cometam erros, como por exemplo, dizerem "vinte e dez" ao invés de trinta, ou transformarem duzentos e dez em "20010".

Os cálculos aritméticos envolvem os fatos aritméticos básicos e os procedimentos para resolução de problemas aritméticos complexos, como por exemplo a subtração, que pode necessitar da passagem de uma coluna para outra. Já os problemas verbais incluem a capacidade de identificar o tipo de problema e transformá-lo em representação matemática (Geary, 2000).

\subsubsection{Contagem}

Para Mix (2010), a contagem verbal exige três habilidades: a criança deve memorizar a sequência de palavras que representa os números (por volta dos três anos, já conta até 10, e aos seis anos, já consegue ir de 10 a 20, de 20 a 30), deve aprender a relacionar a palavra ao objeto, de forma que cada objeto seja contado apenas uma vez e deve entender que o último número contado representa o total de objetos (valor cardinal).

De acordo com Gelman e Gallistel (1978), a contagem é guiada por um conhecimento inato, que os autores denominaram de princípios de contagem, sendo estes a correspondência de um-para-um (entendimento de que cada objeto é contado apenas uma vez); ordem estável (uso dos numerais em ordem fixa); cardinalidade (a quantidade de objetos significa o último numeral falado); irrelevância da ordem de contagem (qualquer ordem de realizar a contagem não altera o resultado); e generalização (o mesmo princípio de contagem para conjuntos de quaisquer elementos).

No entanto, a característica inata desse conhecimento é questionada por estudos apresentados por Fuson e colaboradores (1988) e por Wynn (1992), sugerem que as crianças, inicialmente, não apresentam a cardinalidade, ou seja, não sabem o resultado final da contagem representa o total de elementos, cometem erros durante a contagem e não respeitam a correspondência de um-para-um, não apontando todos os objetos durante a contagem ou passando o dedo correndo pelos objetos enquanto dizem a sequência numérica, sem, no entanto, estabelecer a relação de um-para-um. Ainda 
de acordo com Wynn (1992), até antes dos quatros anos, os diversos números são interpretados pelas crianças apenas como "maiores que 1"; somente a partir dessa idade, e com o envolvimento em situações de contagem, é que elas vão entendendo o número "2", "3", e assim por diante, com a ideia de que sempre se acrescenta uma unidade no número anterior.

Assim, segundo as autoras, a contagem não apresenta um sentindo inato, sendo inicialmente apenas a repetição de palavras sem sentido numérico algum. Esse sentido só é desenvolvido com a participação das crianças em situações onde a contagem é exigida. E ainda, para Wynn (1992), essa aprendizagem é facilitada pelo senso numérico que a criança já possui.

Segundo Barbosa (2007, p.185) "das representações quantitativas iniciais para a contagem verbal ocorre um longo e complexo processo de desenvolvimento". Assim, embora crianças de dois anos consigam identificar diferenças numéricas, elas ainda não sabem o significado de "dois" e "três", e levará um certo tempo para que consigam relacionar essa sequência numérica com a quantidade de elementos de um conjunto. Segundo a autora, o ato de contar é cultural e começa a ser desenvolvido aos dois anos de idade, a partir da interação da criança com seu ambiente social (Barbosa, 2007).

Segundo Lorenzato (2006), contar, para crianças pequenas, pode não ser tão simples quanto parece. Em um primeiro estágio, a criança manipula os objetos e verbaliza numerais em ordem aleatória; por conseguinte, utiliza os dedos para tocar cada objeto, falando o numeral correspondente. Esse processo evolui para contagem de um em um sem utilizar dedos, depois para movimento com os olhos, por subgrupos, e em uma fase mais avança, pode acontecer por multiplicação (Lorenzato, 2006).

Sperafico (2014) acentua que, para o desenvolvimento de todo conhecimento matemático, a contagem é uma habilidade essencial, sobretudo para estabelecer relações entre objeto e representações numéricas. De acordo com o autor, os alunos que desenvolvem melhor essa capacidade, terão melhor desempenho e facilidade em realizar cálculos simples e complexos.

\subsubsection{Processamento Numérico e Cálculo: O Modelo de McCloskey, Caramazza e Basili (1985)}

De acordo com o modelo proposto por McCloskey, Caramazza e Basili (1985), as habilidades matemáticas secundárias se dividem em dois sistemas: o sistema de processamento numérico e o sistema de cálculo. O sistema de processamento numérico inclui os mecanismos de compreensão numérica e produção numérica, enquanto o sistema de cálculo consiste em fatos e procedimentos necessários para a realização dos cálculos.

Nos distintos processos de compreensão e produção numérica, existem componentes para processar numerais arábicos (dígitos) e para números verbais (lidos ou escritos), que possuem, ambos, componentes de processamento lexical e componentes de processamento sintático.

O processamento lexical envolve compreensão ou produção de elementos individuais em um número, como por exemplo, dígito 3 ou a palavra "três". Já o processamento sintático envolve o processamento de relações entre elementos a fim de compreender ou produzir números como um todo, como por exemplo 4759, usando a posição de cada número para determinar o milhar, a centena, a dezena e a 
unidade. Nesse mesmo exemplo, o processamento lexical forneceria apenas o significado dos números individualmente, como "4", "7", "5", "9" (McCloskey, Caramazza, Basili, 1985)

O mesmo processo se dá com os números verbais, mas, no entanto, no processamento lexical dos números verbais, é possível identificar, ainda, os componentes para compreensão ou produção de números falados (componentes de processamento fonológico) e para números escritos (componentes de processamento grafêmico) (McCloeskey, Caramazza, Basili, 1985).

O sistema de cálculo apresenta três componentes principais: 1) mecanismo de processamento de operação símbolo/palavra, para processar símbolos operacionais $(+,-, \times, \div$ ) ou palavras (mais, menos, soma, divisão), que identificam a operação que deve ser realizada; 2) armazenamento de fatos aritméticos, que permite a recordação de fatos aritméticos básicos, como por exemplo a tabuada; e 3) procedimentos de cálculo, como por exemplo, a execução da conta, começando da coluna da direita, escrevendo a soma dos dígitos na parte inferior da coluna, transferindo um para a coluna à esquerda caso o resultado seja maior que nove, etc. (McCloskey, Caramazza, Basili, 1985). Assim, o cálculo exige mecanismos cognitivos específicos, além dos mecanismos de processamento numérico já descritos.

De acordo com Santos (2015), o cálculo é desenvolvido de forma mais tardia na criança, necessita do ensino formal e envolve também a memória de longo prazo.

Em uma revisão realizada por Costa, Rohde e Dorneles (2012) são apresentadas as principais estratégias de contagem, utilizadas pelas crianças para realizar cálculos simples, como o contar todos, contar a partir do primeiro ou da parcela maior.

Segundo Geary (1993) as crianças mais novas utilizam a estratégia de contar todos, e precisam representar as duas parcelas nas mãos, para depois contar todos os dedos; por exemplo, em 3+5, ela contaria 3 em uma das mãos, 5 em outra, e depois contaria os dedos das duas mãos, para que obtivesse o resultado 8. No final da primeira série (do inglês, first grade), as crianças passam a contar a partir do primeiro, quando ela percebe que pode começar da primeira parcela (por exemplo, em $3+5$, ela já iniciaria do 3) e contar a partir do maior, quando iniciam a contagem pela parcela maior, por exemplo, no mesmo cálculo citado anteriormente, inicia-se a partir do 5 e acrescenta-se a parcela menor. Nessas situações, as crianças utilizam, inicialmente, os dedos, materiais manipuláveis e, posteriormente, a contagem verbal e a contagem silenciosa.

Recuperar de forma automática e precisa fatos aritméticos da memória de longo prazo requer uma prática contínua, além do conhecimento conceitual de número e suas relações.

Os progressos para se aprender uma combinação básica são relacionados a três fases: a primeira relaciona-se a estratégias de contagem (ao usar objetos ou contagem verbal para determinar respostas); a segunda fase consiste em estratégias de raciocínio e decomposição (usando fatos conhecidos e relação para deduzir a resposta de uma combinação desconhecida, como por exemplo: $8+6$, decompõe 8 em 6 mais 2, ao somar $6+6=12$, adiciona-se mais 2 ). E por último a fase de domínio, que são processos cognitivos inconscientes ou automáticos, relativamente rápidos (Geary, Hoard, 2005). Na recuperação direta, as crianças declaram uma resposta que está associada a memória de longo prazo especificamente para fatos aritméticos, como ao solicitar $5+3$, as crianças declaram 8. 
A medida que a criança amadurece, passa a resolver problemas mais rapidamente; com a prática, diminui o tempo para executar cada estratégia. Portanto, a recuperação automática desses fatos básicos facilita a resolução de problemas mais complexos (Geary, Hoard, 2005).

Essas habilidades são importantes, pois a criança que memoriza combinações básicas obtém sucesso com a Matemática escolar mais avançada. Acessar diretamente o conhecimento da combinação de números facilita a resolução de problemas, e quando há dificuldades na aritmética, acredita-se que há uma dificuldade de recuperação de fatos na memória de longo prazo (Baroody, Bajwa, Eiland, 2009; Cirino et al., 2007).

Crianças que até o final da primeira série ainda não dominam combinações simples de adição são altamente suscetíveis a dificuldades em Matemática, e também a fracassos e frustrações (Baroody, Bajwa, Eiland, 2009).

Assim, entendemos que a Matemática não é algo tão simples, sendo que seus mecanismos cognitivos incluem o senso numérico, o conhecimento do sistema numérico, representação simbólica e não simbólica, comparar e estimar quantidades, processos fonológicos, inteligência, velocidade de processamento, memória de trabalho e funções executivas, e podem ser afetadas pelas estratégias que se usam para o ensino (Haase, 2014).

\section{O MODELO DE DESENVOLVIMENTO DA COGNIÇÃO NUMÉRICA DE VON ASTER E SHALEV}

No ano de 2007, von Aster e Shalev, descreveram o desenvolvimento da cognição numérica em quatro passos, denominado modelo de Desenvolvimento da Cognição Numérica (von Aster, Shalev, 2007).

O passo 1- Cardinal, ocorre durante a infância, e consiste na compreensão básica de quantidade; é inato, e relacionado a funções adjacentes como a subitização e aproximação. 0 passo 2- Verbal, é o momento em que se estabelece a relação entre a palavra falada ou escrita com a quantidade de objetos ou eventos. O passo 3- Arábico, consiste na relação do símbolo numérico e sua representação da quantidade. O Passo 4 - Ordinal, acontece quando o sistema métrico se estrutura na mente de uma forma contínua, que aumenta gradualmente durante a vida escolar e também adulta (von Aster. Shalev, 2007; Molina, Ribeiro, Santos, von Aster, 2015; Ribeiro, Silva, Santos, 2016).

As crianças pré-escolares, já são dotadas dessas habilidades descritas nos passos 1 e 2, ou seja, 0 senso numérico, que posteriormente subsidiará a aquisição das habilidades secundárias. Na préescola, espera-se que as crianças desenvolvam a contagem de, no mínimo, 10 objetos, que inclui o passo 1, e conceitos simples de adição e subtração, contribuindo gradualmente para o aprendizado do passo 2. Posteriormente, com a entrada da criança no ensino formal, as habilidades mais complexas descritas nos passos 3 e 4 serão adquiridas. No entanto, de acordo com Molina, Ribeiro, Santos e von Aster (2015) as crianças já possuem elementos rudimentares de cada sistema ainda na fase pré-escolar.

\section{O MODELO DO TRIPLO CÓDIGO DE STANILAS DEHAENE}

Segundo Dehaene (1997), a Neurociência pode demonstrar, a partir dos estudos com neuroimagem funcional, que quando o cérebro é confrontado com uma tarefa para o qual não foi preparado, como 
por exemplo a multiplicação com mais de um dígito, ele recruta uma vasta rede de áreas cerebrais. Para ele, como o cérebro humano não possui uma unidade aritmética geneticamente determinada (apenas um senso numérico), ele precisa desenvolver circuitos alternativos para esse fim, que são longos e podem não funcionar bem.

As atividades matemáticas que utilizamos em nossa cultura exigem o recrutamento e a adaptação de vários circuitos nervosos que, embora não sejam programados geneticamente para os processos matemáticos, passam a executar essas funções de forma integrada com os circuitos que originalmente lidam com a noção de quantidade (Cosenza, Guerra, 2011, p.111-112)

Em 1992, Dehaene propôs um modelo que denominou triplo código, capaz de explicar como se dá a manipulação de números pelo cérebro. Para Dehaene e Cohen (1995), as informações numéricas podem ser manipuladas mentalmente de três maneiras: a primeira é a forma numérica visual arábica, que é uma representação interna de dígitos, como por exemplo, 52. A segunda maneira denominase quadro de forma verbal (verbal word framer), no qual os números são representados em uma sequência de palavras; é neste nível que o número cinquenta e dois pode ser notado como cinco dezenas e duas unidades. Nesses níveis, ainda, não há qualquer informação semântica, que se dará somente no terceiro nível, sendo a representação analógica de magnitudes, em que a quantidade e a magnitude são relacionados ao número dado. A representação da magnitude pode ser mostrada em uma linha numérica orientada.

As informações são decodificadas de um código para o outro, e assim, mentalmente pode-se converter um dígito arábico (8) em uma palavra (oito), sem passar pela representação semântica de quantidade (Dehaene, Cohen, 1995; Ciasca, Rodrigues, Azoni, Lima, 2015).

A partir de exames de neuroimagem, é possível identificar as diferentes regiões cerebrais envolvidas nesses diferentes tipos de processamento (Schmithorst, Brown, 2004). Para Dehane (2003), o sistema intraparietal bilateral está associado ao núcleo de sistema de quantidade. As áreas perisilvianas da linguagem no hemisfério esquerdo, principalmente no giro angular, são responsáveis pelo processamento de numerais verbais. As áreas temporo-parieto-occipitais bilaterais, principalmente o giro fusiforme, são responsáveis pelo processamento dos numerais arábicos.

É no corpo caloso que ocorrem as conexões entre as representações analógicas e arábicas de ambos hemisférios. Os aspectos estratégicos de processamento numérico e cálculo dependem das regiões médio-dorsais e dorsolaterais do córtex frontal (Santos, Andrade, Orlando, 2015; Schimithorst, Brown, 2004, Malloy-Diniz et al, 2010).

Os fatos aritméticos envolvem os núcleos da base e regiões do lobo temporal medial, resultando na criação de um domínio específico da memória semântica (Malloy-Diniz et al, 2010).

De forma resumida, segundo Cosenza e Guerra (2011), a noção de quantidade relaciona-se com o lobo temporal, o hemisfério esquerdo calcula enquanto o direito faz estimativas, ambos os hemisférios identificam e comparam números, mas só o hemisfério esquerdo é capaz de decodificar a representação verbal dos algarismos. 


\section{MODELO DE PROCESSAMENTO DE INFORMAÇÃO ARITMÉTICA DE MENON (2010)}

De acordo com a proposta de Menon (2010), o processamento da informação matemática ocorre em três níveis. O nível 1, "Processamento Numérico Básico", consiste na compreensão das propriedades numéricas, incluindo conhecimento dos números, símbolos, noções de quantidade e magnitude, sendo uma estrutura básica sobre a qual desenvolve a aritmética. O nível 2, denominado "Computação Matemática Simples", inclui habilidades de proficiência aritmética, como o cálculo e recuperação da informação do sistema de memória de longo prazo, e desenvolve-se somente após o aprendizado de fatos aritméticos básicos (Dias, Seabra, 2013). O nível 3, "Computação Matemática Complexa", exige outras habilidades como atenção, sequenciamento, tomadas de decisões que influenciam na velocidade e exatidão na realização das tarefas aritméticas.

Segundo Menon (2010), estudos de neuroimagem funcional têm demonstrado as áreas cerebrais envolvidas nesse processamento, sendo o córtex parietal posterior relacionado ao processamento numérico e à recuperação de fatos, enquanto o córtex pré-frontal está implicado na tomada de decisões, sequenciação, memória de trabalho e atenção necessária para recuperar fatos aprendidos e realizar cálculos mais elaborados (MENON, 2010).

\section{CONSIDERAÇÕES}

A Matemática está presente na vida do ser humano desde o nascimento, e mesmo dotado de uma Matemática inata e simples, é no processo de escolarização que são desenvolvidas as habilidades mais complexas, presentes nos adultos.

Fatores biológicos, cognitivos, educacionais e culturais influenciam a cognição numérica, que se constitui de um sistema primário, o senso numérico, e um sistema secundário, que inclui o Processamento Numérico, subdividido em Compreensão e Produção numérica, e o Cálculo.

A compreensão da cognição numérica é fundamental para o professor que atua na Educação Infantil, para melhorar sua prática de ensino e também intervir de maneira adequada quando se observa dificuldades na aprendizagem da Matemática. Quando o professor tem ciência de como a cognição numérica se desenvolve, percebe a importância do desenvolvimento de determinadas habilidades mais básicas pela criança, como base para habilidades mais complexas e torna-se mais capaz de pensar em atividades que podem favorecer o seu desenvolvimento.

As habilidades quantitativas se desenvolvem a partir de capacidades inatas básicas da criança e evolui com a idade, e também por meio da educação formal (Santos et al, 2010, von Aster, 2007). Em um processo de escolarização que tem supervalorizado a alfabetização, a contagem verbal e a escrita dos numerais pode ser um dos principais objetivos, mesmo que a criança ainda não consiga estabelecer relação dessas palavras com as quantidades. Nesse sentido, o conhecimento sobre a Cognição Numérica favorece a discriminação das habilidades básicas necessárias para a compreensão da Matemática mais complexa, e proporciona ao professor embasamento para o desenvolvimento de estratégias que auxiliem os alunos na superação de suas dificuldades.

Não há dúvidas sobre a necessidade de maiores estudos sobre a cognição numérica, sobretudo por parte dos professores, para favorecer o desenvolvimento de habilidades matemáticas e até mesmo, 
contribuir para aprendizagem de crianças com dificuldades, e tornar a aprendizagem da Matemática mais prazerosa.

\section{REFERÊNCIAS}

ANTELL. S. K. D. P. Perception of Numerical Invariance in Neonates. Child Development, v. 54, n. 3, p. 695-701, jun 1983.

BARBOSA, Heloíza Helena de Jesus. Sentido de número na infância: uma interconexão dinâmica entre conceitos e procedimentos. Paidéia. v. 17, n. 37, p. 181 - 194.

BARBOSA, Heloíza Helena de Jesus. Das competências quantitativas iniciais para o conceito de número natural: quais as trilhas possíveis? Psicologia: Reflexão e Crítica, Porto Alegre, v. 25, n. 2, p. 350358, 2012.

BAROODY, A. J., BAJWA, N. P.; EILAND, M. Why Can't Johnny Remember the Basic Facts? Developmental Disabilities Research Reviews, Hoboken, NJ, US, v. 1, n. 15, p. 69-79, Feb. 2009.

BLANCO, Marília Bazan. et al. Uma introdução ao estudo do desenvolvimento das habilidades numéricas. Perspectivas da Educação Matemática, Campo Grande, v. 5, n. 9, p.91-106, jan. 2012.

BRASIL. Ministério da Educação e do Desporto. Secretaria de Educação Fundamental. Referencial curricular nacional para a educação infantil / Ministério da Educação e do Desporto, Secretaria de Educação Fundamental. - Brasília: MEC/SEF, 1998.

CARMO, João do Santos. Comportamento conceitual numérico: um modelo de rede de relações equivalentes (Tese de Doutorado não publicada). Universidade Federal de São Carlos, São Carlos, SP, 2002.

CASE, Robbie. Psychological model of number sense and its development. Paper presented at the annual meeting of the American Educational Research Association, San Diego, Califórnia 1998.

CEZAROTTO, Matheys Araújo. Recomendações para o design de jogos, enquanto intervenções motivadores para crianças com discalculia do desenvolvimento. 2016. $188 \mathrm{f}$. Dissertação (Mestrado em Design) - Universidade Federal do Paraná, Curitiba, 2016.

CIASCA, Sylvia Maria. Revista de Dificuldades. (2004). Disponível em:<http://www.sinprorio.org.br/download/revista/revistadificuldades.pdf>. Acesso em: 14 abr. 2017.

CIRINO, Paul. et al. Errors in Multi-Digit Arithmetic and Behavioral Inattention in Children With Math Difficulties. Journal Of Learning Disabilities, Houston, v. 42, n. 4, p.356-371, July 2009. July/august.

CLEARFIELD, Melissa Wechsler.; MIX, Kelly S. Number Versus Contour Length in Infants: Discrimination of Small Visual Sets. Psychological Science: Research Report, Bloomington, v. 10, n. 5, p.408-411, set. 1999.

COLE. Michael L; COLE, Sheila. R. O Desenvolvimento da Criança e do Adolescente. 4a ed. Porto Alegre, 2003. 
CORSO, Luciana Vellinho; DORNELES, Beatriz Vargas. Senso Numérico e Dificuldades de Aprendizagem na Matemática. Revista de Psicopedagogia, Rio Grande do Sul, v. 27, n. 33, p.298309, jan. 2010.

COSENZA, Ramon M. GUERRA, Leonor B.; Neurociência e Educação. Como o cérebro aprende. Artimed. Porto Alegre, 2011.

COSTA, Adriana Corrêa; ROHDE, Luiz Augusto; DORNELES, Beatriz Vargas. Desenvolvimento de Fatos Numéricos em Estudantes com Transtornos de Aprendizagem. Bolema, Rio Claro, v. 26, n. 44, p.1151-1169, dez. 2012.

DEHAENE, Stanilas. Varieties of numerical abilities. Elsevier Science Publishers B.v. All Rigths Reserved, Paris, v. 1, n. 44, p.1-42, jan. 1992.

DEHAENE, Stanilas.; COHEN, Laurent. Towards an Anatomical and Functional Model of Number Processing. Paris: Lawrence Erilbaum Associates Limited, 1995. 38p.

DEHAENE, Stanilas. The Number Sense: How the mind creates Mathematics. Oxford University Press. 1997.

DEHAENE, Stanilas. Précis of the Number Sense. Mind \& Language. v. 16 n. 1. fev. 2001, p. 16-36.

DEHAENE, Stanilas. et al. THREE PARIETALCIRCUITS FORNUMBER PROCESSING. Cognitive Neuropsychology, Orsay,France, v. 5, n. 20, p.487-506, jan. 2003.

DIAS, Natália Martins.; SEABRA, Alessandra Gotuzo. Competência aritmética sob a perspectiva do processamento da informação: compreensão, desenvolvimento e subsídios para a avaliação. In: SEABRA, Alessandra Gotuzo; DIAS, Natália Martins; CAPOVILLA, Fernando César. Avaliação neuropsicológica cognitiva: leitura, escrita e aritmética, volume 3. São Paulo, Menon, p. 76 $-84,2010$.

FUSON, Karen C. Children's counting and conceptes of number. Springer-Verlag, New York, 1988.

GEARY, David C. Mathematical disabilities: cognitive, neuropsycholigical, and genetc componentes. Psychological bulletin. v. 2, n. 114, 1993.

GEARY, David C. Fom infancy to adulthood: the development of numerical abilities. Europe Child \& Adolescent Psychiatry, Columbia, v. 1, n. 9, p.11-16, jan. 2000.

GEARY, David C.; HOARD, Mary K. Learning Desabilities in arithmetic and mathematics: Theorical and empirical perspectives. In: J. I. D.Handbook of mathematic cognition. New York: Psycology Press, p. 253-267, 2005.

GELMAN, Raquel; GALLISTEL, C. R. The child's understanding of number. Cambridge. 1978. 260 p.

GREEN, Gina. A tecnologia de controle de estímulo no ensino de equivalências número quantidade. In: CARMO, João dos Santos; PRADO, Paulo Sergio Teixeira (Orgs). Relações simbólicas e aprendizagem da matemática. Santo André: ESETec, 2010.

GUIMARÃES, Célia Maria. Perspectiva para Educação Infantil.1a ed. Araraquara: 2005, 236p. 
HAUSER, M., MACNEILAGE, P., WARE, M. Representations in primates: numercial or perceptual? Proceeding of the National Academy of Sciences, v. 93, p. $1514-1517,1996$.

HAUSER, Marc; SPELKE, Elizabeth. Evolutionary and developmental fundations of human knowledge In:GAZZANICA, M. The Cognitive Neuroscience, III. MIT Press, Cambridge, 2004.

LORENA, Angela Bernardo de; CASTRO-CONEGUIM, Janaina de Fátima; CARMO, João dos Santos. Habilidades numéricas básicas: Algumas contribuições da análise do comportamento. Estudos de Psicologia, São Carlos: v. 3, n. 18, p.439-446, jul. 2013.

LORENZATO, Sergio. Educação infantil e percepções matemática. Campinas, SP: Autores Associados, 2006. 197 p.

MALLOY-DINIZ, Leandro F. et al (Org.). Avaliação Neuropsicológica. Porto Alegre: Artimed, 2010. MARCILESE, Mercedes. Aquisição da linguagem e habilidades cognitivas superiores: o papel da língua no desenvolvimento da cognição numérica. Alfa, São Paulo, v. 56, n. 2, p.557-581, jan. 2012.

MCCLOSKEY. M., CARAMAZZA, A., BASILI, A. Cognitive Mechanism in Number Processing and Calculation: Evidence from Dyscalculia. Brain and Cognition, v. 4, p. 171 - 196, 1985.

MENON, V. Developmental cognitive neuroscience of arithmetic: implications for learning and education. Mathematics Education, 42, 515-525. 2010.

MIX, Kelly S. Habilidades iniciais em operações com números: a transição dos primeiros meses de vida até a primeira infância. Enciclopédia Sobre o Desenvolvimento na Primeira Infância, Michigan, v. 1, n. 1, p.1-5, jun. 2010.

MOLINA, Juliana. et al. Cognição numérica de crianças pré-escolares brasileiras pela ZAREKIK. Temas em Psicologia, Bauru, v. 23, n. 1, p.123-135, 2015.

PAPALYA, Diane. E., OLDS, Sally. W, FELDMAN, Ruth D. O mundo da criança. Da infância à adolescência. $11^{\circ}$ ed. Porto Alegre: AMGH, 2010.so, revisão técnica: Maria Cecília de Vilhena Moraes Silva, Odete de Godoy Pinheiro - $11^{\circ}$ ed. Dados eletrônicos - Porto Alegre: AMGH, 2010. 610 p.

QEDU. Use dados e transforme a educação. 2017. Disponível em: <http://www.qedu.org.br>. Acesso em: 28 mar. 2017.

RIBEIRO, Fabiana Silva; SILVA, Paulo Adilson da; SANTOS, Flavia Heloísa dos. Padrões de dissociação da Memória Operacional na Discalculia do Desenvolvimento. In: SALLES, Jerusa Fumagalli de; HAASE, Vitor Geraldi.; MALLOY-DINIZ, Leandro F. Neuropsicologia do Desenvolvimento: Infância e adolescência. Porto Alegre: Artimed, 2016. Cap. 15, p. s/p.

RODRIGUES, Sonia das; RIECHI; T. I. J. de S. Discalculia do Desenvolvimento. In: CIASCA, Sylvia M.; RODRIGUES, Sonia das; AZONI, Cíntia A. S. LIMA, Ricardo F. de. Transtornos Aprendizagem: Neurociência e interdisciplinaridade. Ribeirão Preto: Book Toy, 2015. p. 239-247.

SANTOS, Flavia Heloisa dos; et al. Novos instrumentos para avaliação de habilidades matemáticas em crianças. In: V Congresso Brasileiro de Tecnologia e (Re) Habilitação Cognitiva. São Paulo, Brasil, 2006. 
SANTOS; Flavia Heloisa dos S.; et al. Recomendações para professores sobre o transtorno da matemática. O desafio de educar. Lidando com os problemas na aprendizagem e no comportamento. Sinpro-Rio. Rio de Janeiro e Região. n.5, p. 19-31, mai. 2010.

SANTOS, Flavia Heloísa dos, et al. Cognição Numérica: Contribuições à Pesquisa Clínica. In: PRADO, P. S. T. do, CARMO, J. dos S. (Org.). Diálogos sobre ensino-aprendizagem da matemática. Abordagens pedagógica e neuropsicológica. São Paulo. Cultura Acadêmica. 2016. P.63-91.

SEABRA, Alessandra; DIAS, Natália Martins; MACEDO, Elizeu Coutinho. Desenvolvimento das habilidades aritméticas e composição fatorial da prova de Aritmética em estudantes do Ensino Fundamental. Interamerican Journal of Psychology, 44 (3), p.481-488, 2010.

SCHMITHORST, Vincent J.; BROWN, Rhonda Douglas. Empirical validation of the triple-code model of numerical processing for complex math operations using functional MRI and group Independent Component Analysis of the mental addition and substraction of fractions. Neuroimage, Cincinnati, $v$. 1, n. 22, p.1414-1420, maio 2004.

SPELK, Elizabeth; KINZLER, Katherine D.; Core Knowledge. Developmental Science. USA. V.10, n.1, p. 89-96, 2007.

SILVA, Paulo Adilson da; RIBEIRO, Fabiana Silva; SANTOS, Flavia Heloísa dos. Cognição numérica em crianças com transtornos específicos de aprendizagem. Temas em Psicologia, Bauru, v. 23, n. 1, p.197-210, 2015. Associação Brasileira de Psicologia. Disponível em: <http://dx.doi.org/>. Acesso em: 18 jan.2017.

SPERAFICO, Yasmini Lais Spindler. Intervenção no uso de procedimentos e estratégias de contagem com alunos dos anos iniciais com baixo desempenho em matemática. Revista de Psicopedagogia, Petrópolis - Porto Alegre, v. 94, n. 31, p.11-20, jan. 2014.

VON ASTER, Michael G.; SHALEV, Ruth S. Number development and developmental dyscalculia. Developmental Medicine \& Child Neurology, Berlin, Germany, n. 49, p.868-873, jan. 2007.

VON ASTER, Michael G; DELLATOLAS, G. ZAREKI-R: Batterie pour l'evaluation du traitement des nombres et du calcul chez l'enfant. Adaptation francaise. Paris: ECPA. 2006.

WEINHOLD-ZULAUF, M.; SCHWEITER, M.; VON ASTER, Michael. Das kindergartenalter: sensitive periode für die entwicklung numerischer fertigkeiten. Kindheit und Entwicklung, v. 12, p. 222-230, 2003.

WYNN, Keating. Addition and subtration bu human infants. Nature, $358-750$ 